\title{
Quantifying quantum-amplified metrology via Fisher information
}

\author{
G. S. Agarwal $\oplus^{1,2}$ and L. Davidovich $\oplus^{1,3}$ \\ ${ }^{1}$ Institute for Quantum Science and Engineering, Texas A\&M University, College Station, Texas 77843, USA \\ ${ }^{2}$ Departments of Biological and Agricultural Engineering and Physics and Astronomy, \\ Texas A\&M University, College Station, Texas 77843, USA \\ ${ }^{3}$ Instituto de Física, Universidade Federal do Rio de Janeiro, Rio de Janeiro, RJ 21941-972, Brazil
}

(Received 10 November 2021; accepted 21 January 2022; published 9 February 2022)

\begin{abstract}
We present a unified framework, based on quantum metrology concepts, for defining and quantifying deterministic noiseless quantum amplification of parameter-dependent processes, which plays an important role in increasing the precision of quantum sensing. Recent experiments [Burd et al., Science 364, 1163 (2019)] can be encompassed by this concept, which also leads to new suggestions of experimental work. The unified view presented here allows the identification of the basic steps for quantum amplification and of the measurements that lead to the best possible precision, beyond the quantum standard limit, in the estimation of parameters involved in the process. This is applied to the estimation of displacements of trapped ions and of the phase in $\operatorname{SU}(1,1)$ optical interferometers and atomic interferometry.
\end{abstract}

DOI: 10.1103/PhysRevResearch.4.L012014

\section{INTRODUCTION}

Quantum linear phase-preserving amplifiers necessarily introduce noise [1,2], which can be seen as a consequence of the no-cloning theorem $[3,4]$. Based on ideas from probabilistic quantum cloning [5], nondeterministic noiseless amplification $[6,7]$ has been proposed to circumvent this restriction. On the other hand, it is possible to have noiseless deterministic phasesensitive quantum amplification [2,8], which may play an important role in space communication [9]. Another instance of noiseless deterministic quantum amplification is the magnification of parameters involved in dynamical processes, such as the displacement of an ion [10], due to an applied force, the phase in an optical or atomic interferometer [11], or the amplitude of a small microwave field injected into a cavity [12]. In this case, the deterministic and noiseless amplification allows the estimation of parameters that can be otherwise too small to be detected. Here we present a unified framework, based on quantum metrology concepts, for defining and quantifying deterministic noiseless quantum amplification of parameterdependent processes. Recent experiments can be analyzed within this framework, which also leads to new suggestions of experimental work, through the identification of the basic steps for quantum amplification and of the measurements that lead to the best possible precision in the estimation of the relevant parameters. The amplification process is an important tool for quantum advantage in parameter estimation. We apply these ideas to the estimation of the displacement of a trapped

Published by the American Physical Society under the terms of the Creative Commons Attribution 4.0 International license. Further distribution of this work must maintain attribution to the author(s) and the published article's title, journal citation, and DOI. ion and of the phase in a $\mathrm{SU}(1,1)$ interferometer, as well as to atomic interferometry.

The increase in precision in the estimation of parameters in the last few years has led to sophisticated technological and scientific achievements, exemplified by the amazing detection of gravitational waves at LIGO [13], involving the estimation of a change in the lengths of the arms of an interferometer of the order of $1 / 10000$ of the radius of a proton; new portable quantum gravimeters [14], which use atomic interference of matter waves to measure the local value of gravitational acceleration, achieving sensitivities of the order of one-trillionth of the Earth surface gravity; quantum accelerometers and speedometers [15], allowing GPS-free navigation; atomic clocks with accuracy at the $10^{-18}$ level [16]; and the measurement of very weak electric fields, which, with increasing precision, should enable the use of trapped ions in the search for dark matter [17].

Quantum metrology involves the use of quantum states to increase the precision in the estimation of parameters that characterize physical processes. It allows for the precise quantification of the uncertainty in the estimation of parameters, through a sequence of steps that involve (i) the preparation of a probe in a suitable initial state, (ii) the interaction of the probe with the process under investigation, (iii) a suitable measurement of the final state of the probe, and (iv) the association of each experimental result with an estimator, which leads to the quantification of the uncertainty. Identification of the best state of the incoming probe and the best measurement procedure is usually a tough challenge. For unitary evolution, it is possible to find analytical solutions [18-20], which are, however, not available in general for open systems, except for some specific examples [21-25], while numerical solutions, although useful, become cumbersome for high-dimensional Hilbert spaces. It is known, in fact, that preferred states for estimating parameters may become practically useless when losses are present [26]. 
The basic machinery for tackling parameter estimation was formulated, within the classical framework, by Fisher [27], Cramér [28], and Rao [29]: For a complete set of experimental results $\{j\}$, obtained through measurements on a probe that carries information about the parameter $x$ to be estimated, and for unbiased estimators, so that the estimation of the parameter, averaged over all experimental results, $\langle x\rangle$, coincides with the true value of $x$ and $d\langle x\rangle / d x=1$ (implying that the average also coincides with the value of the parameter in an infinitesimal neighborhood of $x$ ), the lower bound for the standard deviation in the estimation of $x$ is given by the Cramér-Rao expression,

$$
\Delta x \geqslant \frac{1}{\sqrt{\mathcal{N} F(x)}},
$$

where $\mathcal{N}$ is the number of repetitions of the experiment and $F(x)$ is the Fisher information, given by

$$
F(x)=\sum_{j} \frac{1}{P_{j}(x)}\left[\frac{d P_{j}(x)}{d x}\right]^{2},
$$

with $P_{j}(x)$ being the probability of getting the experimental result $j$ if the value of the parameter is $x$. Fisher showed that under some general conditions (which are those for the applicability of the central limit theorem), this bound is achievable in the limit $\mathcal{N} \rightarrow \infty$. This expression can be immediately generalized to quantum mechanics by expressing the probabilities $P_{j}(x)$ in terms of a positive operator-valued measure (POVM) $\left\{\hat{E}_{j}\right\}$, with $\sum_{j} \hat{E}_{j}=1$, so that $P_{j}(x)=\operatorname{Tr}\left[\hat{\rho}(x) \hat{E}_{j}\right]$, where $\hat{\rho}(x)$ is the density operator corresponding to the outgoing probe. The ultimate precision limit, for a given initial state of the probe, is obtained by maximizing $F(x)$ over all possible measurements, which leads to the quantum Fisher information $\mathcal{F}_{Q}(x)$. For a parameter-dependent unitary evolution $\hat{U}(x)$ of the probe, $\mathcal{F}_{Q}(x)=4(\Delta \hat{\mathcal{G}})^{2}$, where $(\Delta \hat{\mathcal{G}})^{2}$ is the variance, in the initial state of the probe, of the operator $\hat{\mathcal{G}} \equiv i\left[d \hat{U}^{\dagger}(x) / d x\right] \hat{U}(x)$.

In the following, we apply these concepts in order to determine the best possible precision and the best measurement procedure for parameter estimation through quantum deterministic and noiseless amplification of parameter-dependent processes.

\section{ESTIMATING MECHANICAL OSCILLATOR MOTION THROUGH QUANTUM AMPLIFICATION}

Here we consider the quantum amplification of mechanical oscillator motion, as described in [10], which demonstrates a technique for amplifying coherent displacements of a mechanical oscillator with initial magnitudes well below the zero-point fluctuations. The protocol, applied to a trapped-ion mechanical oscillator, involved applying two squeezing operations, one before and the other after a small displacement of the ion, with the second operation undoing the squeezing implemented by the first one.

This sequence of operations, applied to the ground state of the oscillator, can be described by the equation

$$
|\psi\rangle=\hat{S}^{-1}(\xi) \hat{D}(\alpha) \hat{S}(\xi)|0\rangle,
$$

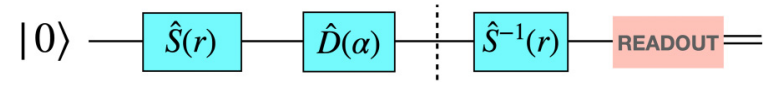

FIG. 1. Circuit for quantum amplification of the displacement of an ion, initially in the ground state, as described in [10]. The initial ground state is squeezed and then displaced. Everything to the right of the dashed line is considered as part of the measurement process. Final detection, after the second squeezing transformation, is done by transferring the information on the displacement to internal degrees of freedom of the ion. The state after the inverse squeezing operator is also a coherent state, with amplified amplitude, so noise is not increased in the process.

where $\hat{D}(\alpha)$ is the displacement operator,

$$
\hat{D}(\alpha)=\exp \left(\alpha \hat{a}^{\dagger}-\alpha^{*} \hat{a}\right),
$$

with $\alpha=|\alpha| \exp (i \phi)$ being the displacement, and

$$
\hat{S}(\xi)=\exp \left[\left(\xi^{*} \hat{a}^{2}-\xi \hat{a}^{\dagger 2}\right) / 2\right]
$$

is the squeezing operator, with squeezing parameter $\xi(r, \theta)=$ $r \exp (i \theta)$. It follows that $|\psi\rangle$ in (3) is also a coherent state $\left|\alpha_{f}\right\rangle$, with $\alpha_{f}=|\alpha|\{\exp (i \phi) \cosh (r)+\exp [i(\theta-$ $\phi)] \sinh (r)\}$. Maximum amplification is obtained when $\phi=$ $\theta=0$, that is, the displacement is along the squeezed axis. One then has

$$
\left|\alpha_{f}\right\rangle=|G \alpha\rangle, \quad G=e^{r},
$$

where $G$ is the amplification factor. Since the final state is also a coherent state, as is the initial state, there is no increase in the quantum noise, which corresponds to the width of the initial ground state. The corresponding quantum circuit is shown in Fig. 1.

In the experiment reported in [10], the information on the motional state of the ion is transferred to a couple of its internal states (denoted by $|\uparrow\rangle$ and $|\downarrow\rangle$ ) within the ${ }^{2} S_{1 / 2}$ electronic ground-state hyperfine manifold, using a phase-sensitive redsideband method. The ion is initialized in the electronic and motional ground state $|\downarrow\rangle|0\rangle$, and the qubit state can be manipulated with a microwave carrier pulse using a magnetic dipole transition. The population of the state $|\downarrow\rangle$ is then measured by applying a laser resonant with the ${ }^{2} S_{1 / 2} \leftrightarrow{ }^{2} P_{3 / 2}$ cycling transition and detecting state-dependent ion fluorescence. The probability of measuring the state $|\downarrow\rangle$ is then

$$
P_{\downarrow}=\frac{1}{2}\left[1-C\left(\left|\alpha_{f}\right|\right) \cos \phi\right],
$$

where $\phi$ is the phase of the carrier $\pi / 2$ pulse, which follows the red-sideband mapping pulse, and $C\left(\left|\alpha_{f}\right|\right)$ is the signal contrast, defined as

$$
C \equiv P_{\downarrow, \max }-P_{\downarrow, \min },
$$

where $P_{\downarrow, \max }$ and $P_{\downarrow, \text { min }}$ are the maximum and minimum, respectively, of the fringes obtained by varying the phase $\phi$, and attained for $\phi=\pi$ (maximum) and $\phi=0$ (minimum). Neglecting decoherence, one has [10]

$$
C\left(\left|\alpha_{f}\right|\right)=2\left|\alpha_{f}\right| f\left(\left|\alpha_{f}\right|\right),
$$

where

$$
f\left(\left|\alpha_{f}\right|\right)=\sum_{n=0}^{\infty} \frac{e^{-\left|\alpha_{f}\right|^{2}}\left|\alpha_{f}\right|^{2 n}}{n !}\left[\frac{\cos \left(\frac{\pi}{2} \sqrt{n}\right) \sin \left(\frac{\pi}{2} \sqrt{n+1}\right)}{\sqrt{n+1}}\right] .
$$


For $G \alpha \ll 1, C\left(\left|\alpha_{f}\right|\right) \approx 2\left|\alpha_{f}\right|=2 G|\alpha|$. In [10], the signal-tonoise ratio (SNR) is calculated as

$$
\mathrm{SNR}=C(G \alpha) / \sigma[C(G \alpha),
$$

where $\sigma[C(G \alpha)]$ is obtained, for $G|\alpha| \ll 1$, from projection noise considerations, which lead to $\sigma \sim 1 / \sqrt{\mathcal{N}}$, with $\mathcal{N}$ being the total number of measurements. In this limit, the state of the final coherent state can be written, up to terms linear in $G \alpha$, as $\left|\alpha_{f}\right\rangle \approx|0\rangle+G \alpha|1\rangle$, which can be considered as a qubit. The SNR is then given by

$$
\mathrm{SNR}=2 G|\alpha| \sqrt{\mathcal{N}} .
$$

This expression displays the amplification of the signal-tonoise ratio, due to the sequence of operations implemented on the initial ground state, which amounts to doing a squeezing transformation and then undoing it, after the displacement. It is indeed a noiseless amplification since the noise comes from the measurement procedure, which involves the transference of the information on the motional state to a couple of internal states of the ion and the following detection of the population of the lower state. In the Appendix, we show how $\sigma$ can be obtained from quantum optics methods.

A natural question that arises from this analysis is what the best possible measurement procedure would be for this kind of amplification process. Here we explore the tools of quantum metrology in order to answer this question.

A key element in this respect is to separate the preparation (the resource state) and the measurement process, as shown in Fig. 1. Clearly, if one wants to reduce the uncertainty in the measurement of the displacement, a state squeezed in the direction of the displacement should be used. This defines the resource state-it is the squeezed state obtained by applying the squeezing transformation (5) to the initial ground state,

$$
|\xi\rangle \equiv|r, \theta\rangle=\hat{S}(r, \theta)|0\rangle .
$$

We consider, for simplicity, that $\alpha$ is real. It follows then that the maximum amplification is obtained for $\phi=\theta=0$, which implies a phase-sensitive amplification. On the other hand, for the displacement operator $\hat{D}=\exp \left[\alpha\left(\hat{a}^{\dagger}-\hat{a}\right)\right]$, the quantum Fisher information is given by four times the variance of the operator $\hat{\mathcal{G}}=i[d \hat{D}(\alpha) / d \alpha] \hat{D}^{\dagger}(\alpha)=i\left(\hat{a}^{\dagger}-\hat{a}\right)$ in the state (13), that is,

$$
\mathcal{F}_{Q}(\alpha)=\left\langle r\left|\Delta^{2} \mathcal{G}\right| r\right\rangle=4 G^{2}, \quad G \equiv e^{r},
$$

where we have used the notation $|r\rangle \equiv|r, 0\rangle$. This implies that for $\mathcal{N}$ experimental realizations, the standard deviation in the estimation of the displacement $\alpha$ is

$$
\Delta \alpha /|\alpha|=\frac{1}{2 G|\alpha| \sqrt{\mathcal{N}}} .
$$

The inverse of this relation coincides with (12). This implies that for $G|\alpha| \ll 1$, the measurement procedure of first squeezing the initial state, then displacing it and reversing the squeezing, and, finally, detecting the qubit to which information on the motional state was transferred attains the ultimate precision limit derived from the quantum Fisher information.

This is not so, however, if the condition $G|\alpha| \ll 1$ is violated. The Fisher information corresponding to the mea-

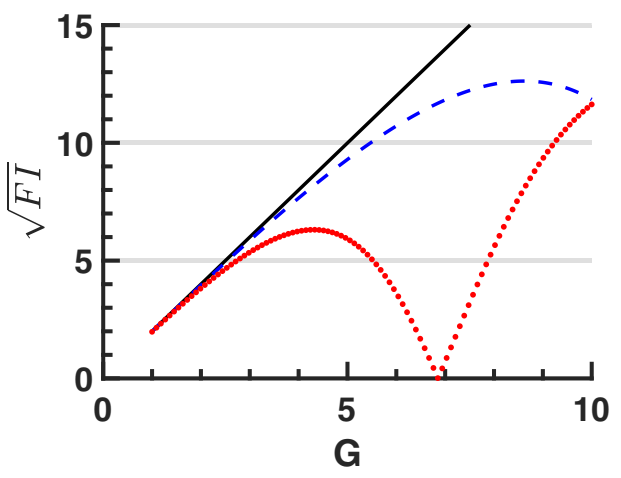

FIG. 2. Square root of the Fisher information corresponding to the detection scheme in [10], as a function of the squeezing factor $G=e^{r}$, for several values of $\alpha: 0.005$ (solid line), 0.05 (dashed line), and 0.1 (dotted line). For $G|\alpha| \ll 1$, it coincides with the value given by the quantum Fisher information: $\sqrt{\mathcal{F}_{Q}}=2 G$. This is not so, however, for higher values of $G|\alpha|$ : estimation of the displacement then requires the measurement of the entire phonon-number distribution, rather than measuring the qubit represented by two internal states of the ion.

surement adopted in [10] can be calculated as

$$
\begin{aligned}
F(\alpha) & =\frac{1}{P_{\downarrow, \text { max }}}\left[\frac{\partial P_{\downarrow, \text { max }}}{\partial \alpha}\right]^{2}+\frac{1}{P_{\downarrow, \text { min }}}\left[\frac{\partial P_{\downarrow, \text { min }}}{\partial \alpha}\right]^{2} \\
& =\frac{1}{P_{\downarrow, \text { max }}\left(1-P_{\downarrow, \text { max }}\right)}\left[\frac{\partial P_{\downarrow, \text { max }}}{\partial \alpha}\right]^{2} \\
& =\frac{G^{2}}{\left[\frac{1}{2}+x f(x)\right]\left[\frac{1}{2}-x f(x)\right]}\left\{\frac{\partial}{\partial x}[x f(x)]\right\}^{2},
\end{aligned}
$$

where, in the last line, $x=G \alpha$ and we used that $P_{\downarrow, \min }=$ $1-P_{\downarrow, \max }$. For $\mathcal{N}$ measurements, the corresponding Fisher information becomes $\mathcal{N} F(\alpha)$. One should note that the projection noise is automatically taken into account in the Fisher information (see second line in the above expression), where, for $\mathcal{N}$ measurements, $P_{\downarrow, \max }\left(1-P_{\downarrow, \max }\right) / \mathcal{N}$ coincides with $\sigma^{2}$ calculated in [10]. This expression is plotted in Fig. 2, as a function of $G$, for several values of $\alpha$. It is clear that as long as $G|\alpha| \ll 1$, the agreement with the quantum Fisher information is excellent. This is due to the fact that for $G|\alpha| \ll 1$, the final coherent state can be represented by the qubit $|0\rangle+G \alpha|1\rangle$, implying that the transcription to the internal atomic qubit does not lose information about the displacement. For higher values of $G|\alpha|$, however, the discrepancy increases since the motional state cannot be reduced to a qubit. Figure 2 shows that the Fisher information corresponding to the qubit measurement may actually vanish, meaning that no information whatsoever is available on the displacement at this point. In fact, it should not be expected that the whole information about the displaced state could be retrieved from a qubit.

This result should be compared with the one obtained through phonon counting. After the second squeezing transformation, the motional phonon-number distribution is

$$
P_{n}(\beta)=\frac{e^{-|\beta|^{2}}}{n !}|\beta|^{2 n}, \quad \beta=G \alpha .
$$


The corresponding Fisher information is

$F=\sum_{n=0}^{\infty} \frac{1}{P_{n}(\alpha)}\left[\frac{\partial P_{n}(\alpha)}{\partial \alpha}\right]^{2}=G^{2} \sum_{n=0}^{\infty} \frac{1}{P_{n}(\beta)}\left[\frac{\partial P_{n}(\beta)}{\partial \beta}\right]^{2}=4 G^{2}$,

which coincides with the quantum Fisher information for all values of $G \alpha$. This implies that phonon counting allows maximal information on the displacement. One should note that the phonon-number distribution is actually addressed in [10], in order to characterize the squeezing operation. On the other hand, the experimental strategy adopted in [10] for $|G \alpha| \ll 1$ seems to be less time consuming than the phonon-counting measurement. However, phonon counting does lead to more information on the displacement since it does not require the restriction to $|G \alpha| \ll 1$.

The idea of implementing a transformation before the displacement of a parameter and then undoing it is present in many other quantum metrological tasks, involving, for instance, phase shifts in interferometers (see Secs. III and IV) and displacements of the electromagnetic field in a mode of a superconducting cavity [12], which is similar to the present example, since the cavity mode can be considered as a harmonic oscillator. While for the experiment in [10] the amplification comes from the squeezing parameter, in [12] the amplification factor is the distance between two coherent states in an entangled atom-field Schrödinger-cat state, produced by the resonant interaction between a Rydberg atom and a coherent state in the cavity. After the displacement, a time-inversion protocol disentangles the atom and the field, and the detection of the atomic state allows the estimation of the displacement, beyond the standard quantum limit.

The strategy of "doing" and "undoing" was reported in [30] for the measurement of phonon frequency fluctuations, through the use of superpositions of phonon-number states, and in [17] for quantum-enhanced sensing of displacements and electric fields.

We consider in the following two further examples: the measurement of phase in SU $(1,1)$ interferometers [31-35] and in atomic interferometry [11,36-38].

\section{MEASUREMENT OF PHASE VIA TWO-MODE SQUEEZED STATES}

We next consider a similar scenario for the measurement of phase via $\operatorname{SU}(1,1)$ interferometry [31]. The scheme is shown in Fig. 3. Here, $\hat{S}_{a b}(\gamma)=\exp \left[\gamma\left(\hat{a}^{\dagger} \hat{b}^{\dagger}-\hat{a} \hat{b}\right)\right]$ is a two-mode squeezing transformation, and $\phi$ is the phase to be estimated. The error sensitivity for this measurement is obtained from the measured signal, $\left\langle\hat{N}_{\text {out }}\right\rangle$, where $\hat{N}_{\text {out }}=\hat{a}_{\text {out }}^{\dagger} \hat{a}_{\text {out }}+\hat{b}_{\text {out }}^{\dagger} \hat{b}_{\text {out }}$, and the fluctuations in $\hat{N}_{\text {out }}$, expressed in terms of the standard deviation $\Delta \hat{N}_{\text {out }}$. The averages here are taken in the initial state. The best result, which yields the minimum value for the phase uncertainty, is found around $\phi=0$ [39]:

$$
\Delta \phi=\frac{\Delta \hat{N}_{\text {out }}}{\partial\left\langle\hat{N}_{\text {out }}\right\rangle / \partial \phi}=\frac{1}{2 \cosh r \sinh r}=\frac{1}{\sqrt{\langle N\rangle(2+\langle N\rangle)}},
$$

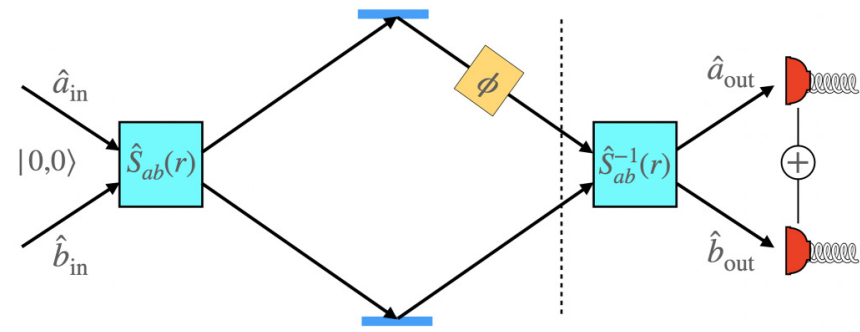

FIG. 3. Phase estimation in a $\mathrm{SU}(1,1)$ interferometer. $\hat{S}_{a b}(r)$ is a two-mode squeezing transformation, applied to the vacuum state of both modes, and $\phi$ is the phase to be estimated, through the detection of the total number of photons emerging from the interferometer. Everything to the right of the dashed line is considered part of the measurement process.

where $\langle N\rangle=2 \sinh ^{2} r$ is the average number of photons in the two-mode vacuum squeezed state, which is the resource in this case. The Heisenberg scaling is obtained when $\langle N\rangle \gg 1$.

From the perspective of the quantum Fisher information, the initial resource state is $\hat{S}_{a b}(r)|0,0\rangle$. The phase shift in the dispersive arm of the interferometer is represented by the unitary operator $e^{i \phi \hat{a}^{\dagger} \hat{a}}$. Thus the quantum Fisher information $\mathcal{F}_{Q}(\phi)$ is $4\left[\left\langle\left(\hat{a}^{\dagger} \hat{a}\right)^{2}\right\rangle-\left\langle\hat{a}^{\dagger} \hat{a}\right\rangle^{2}\right]$, where the expectation value is computed in the state $\hat{S}_{a b}(r)|0,0\rangle$. It is easily seen that $\mathcal{F}_{Q}(\phi)=4\left\langle\hat{a}^{\dagger} \hat{a}\right\rangle\left(1+\left\langle\hat{a}^{\dagger} \hat{a}\right\rangle\right)=4 \sinh ^{2} r \cosh ^{2} r$, leading to (19). Thus, the measurement in Fig. 3 saturates the CramérRao bound. Note that everything to the right of the dashed line in Fig. 3 has to be considered as part of the measurement process. This includes the action of the second inverse squeezing transformation, which, similarly to the previous example, undoes what was done by the first squeezing transformation.

\section{MEASUREMENT OF PHASE IN ATOMIC INTERFEROMETRY}

A similar analysis applies to atomic interferometry with entangled states. Consider the state $|\downarrow \ldots \downarrow\rangle$, containing an even number of qubits characterized by the states $|\downarrow\rangle$ and $|\uparrow\rangle$ (a more general treatment, valid for even and odd states, was considered in [11]). A resource state for phase estimation is obtained by applying to this state the entangling transformation $\hat{U}=\exp \left(i \pi \hat{J}_{x}^{2} / 2\right)[11,40-42]$, where $\hat{J}_{x}$ is the $x$ component of the collective angular momentum operator associated with the $N$ qubits.

The scheme displayed in Fig. 4 allows the quantum amplification of a phase shift undergone by the qubits. The measurement corresponds to the scheme to the right of the dashed line. Again, the quantum amplification process involves "doing" and "undoing": the first entangling transformation, used to build the resource state, is undone after the phase shift.

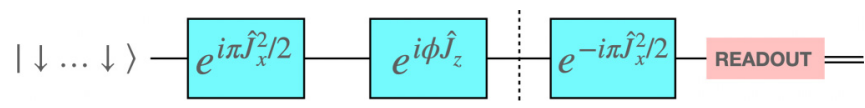

FIG. 4. Phase estimation in atomic interferometry. The operator $\exp \left(i \pi \hat{J}_{x}^{2} / 2\right)$ leads to an entangled state, which probes the phase $\phi$. The inverse operator $\exp \left(-i \pi \hat{J}_{x}^{2} / 2\right)$ allows the estimation of $\phi$ through measurement of the population inversion. 
One should note that the resource state in this case is ([39], Sec. 12.9),

$$
|\psi\rangle=e^{i \pi \hat{\delta}_{x}^{2} / 2}|\downarrow, \ldots \downarrow\rangle=\frac{e^{i \pi / 4}}{\sqrt{2}}\left[|\downarrow \ldots \downarrow\rangle+i^{N-1}|\uparrow \ldots \uparrow\rangle\right] .
$$

The quantum Fisher information corresponding to this resource state and the phase-shift operator $e^{i \phi \hat{f}_{z}}$ is $\mathcal{F}_{Q}(\phi)=$ $4\left(\left\langle\hat{J}_{z}^{2}\right\rangle-\left\langle\hat{J}_{z}\right\rangle^{2}\right)=N^{2}$, yielding a phase uncertainty $\Delta \phi=$ $1 / N$, which corresponds to Heisenberg scaling.

We show now that measuring the population inversion in the final state, after the operator $\exp \left(-i \pi \hat{J}_{x}^{2} / 2\right)$, leads to the bound obtained from the quantum Fisher information. The final state is

$$
\left|\psi_{f}\right\rangle=\cos (N \phi / 2)|\downarrow \ldots \downarrow\rangle+i^{N} \sin (N \phi / 2)|\uparrow \ldots \uparrow\rangle,
$$

so the probabilities of finding the qubits in the states $|\downarrow \ldots \downarrow\rangle$ and $|\uparrow \ldots \uparrow\rangle$ are $p_{\downarrow}=\cos ^{2}(N \phi / 2)$ and $p_{\uparrow}=\sin ^{2}(N \phi / 2)$, respectively. The corresponding Fisher information is then

$$
F(\phi)=\frac{1}{p_{\downarrow}}\left(\frac{\partial p_{\downarrow}}{\partial \phi}\right)^{2}+\frac{1}{p_{\uparrow}}\left(\frac{\partial p_{\uparrow}}{\partial \phi}\right)^{2}=N^{2},
$$

which leads to the bound given by the quantum Fisher information.

One should note that in order to determine $p_{\downarrow}$ and $p_{\uparrow}$, it is sufficient to measure the population of the first qubit in (21). This leads to the best possible value for the lower bound for $\Delta \phi$, which is achieved in the limit of a very large number of measurements (more precisely, when $\mathcal{N} \rightarrow \infty$ ).

This shows that the measuring of the phase in atomic interferometry may also be placed in the framework of doing and undoing of [10] and [12]. An alternative method was discussed in [11]. Instead of decoding the phase information in (20) through a disentangling operation, this was achieved by a parity measurement (odd and even numbers of particles in the state $|\downarrow\rangle)$ after a parity pulse with variable phase, which may be simpler than the disentangling transformation, but has the disadvantage that measurement of the parity is very difficult for large values of $N$, in the presence of noise.

\section{CONCLUSION}

We have shown that the idea of quantum amplification of parameter-dependent processes, experimentally demonstrated in [10], within the context of displaced trapped ions, encompasses a broad class of parameter-estimation situations. It involves applying a transformation that leads to a resource state, which undergoes the parameter-dependent process, and subsequently undoing the transformation, thus decoding the information on the parameter in the state of the probe. The examples discussed here show that this procedure, when associated with the tools of quantum metrology, allows the determination of the best measurement procedure leading to results for the precision in agreement with those obtained from the quantum Fisher information. In particular, we have applied these ideas to $\mathrm{SU}(1,1)$ and atomic interferometers, showing how our approach leads to Heisenberg scaling in the precision. As discussed in Sec. IV, the procedure of doing and undoing is by no means the only possible strategy to reach the best precision. It provides, however, a nice example of noiseless quantum amplification, a unifying concept for a broad class of experiments. In any case, the quantum Fisher information is a useful benchmark for designing experiments leading to the best possible parameter estimation. This was illustrated in connection with the experiment reported in [10], for which phonon counting leads to optimal estimation of the displacement.These findings may motivate further experimental work on these and other systems, aiming at increasing the precision of parameter estimation through the quantumamplification strategy. Due to its generality, our analysis can be applied to a diversity of quantum sensing tasks.

\section{ACKNOWLEDGMENTS}

G.S.A. acknowledges the support of the Air Force Office of Scientific Research (AFOSR Award No. FA9550-20-1-0366) and the Robert A. Welch Foundation (Grant No. A-1943). L.D. acknowledges the support of the Brazilian agencies CNPq and FAPERJ and the Brazilian National Institute of Science and Technology for Quantum Information. The authors thank Jiaxuan Wang for producing Fig. 2.

\section{APPENDIX: QUANTUM OPTICAL DERIVATION OF THE SIGNAL-TO-NOISE RATIO}

In this Appendix, we present a calculation of $\sigma[C(G \alpha)]$ in Eq. (11), using the fluctuations in the operator that corresponds to the contrast $C$, as defined in Eq. (8). In [10], the measured quantity $P_{\downarrow}$ is obtained from

$$
\begin{aligned}
P_{\downarrow} & =\sum_{n}\left|\left\langle n\left|\left\langle\downarrow\left|\hat{R}(\pi / 2, \phi) \hat{U}_{\mathrm{RSB}} D(G \alpha)\right| \downarrow\right\rangle\right| 0\right\rangle\right|^{2} \\
& =\left\langle G \alpha\left|\left\langle\downarrow\left|\hat{U}_{R S B}^{\dagger}\right| \psi_{\downarrow}\right\rangle\left\langle\psi_{\downarrow}\left|\hat{U}_{\mathrm{RSB}}\right| G \alpha\right\rangle\right| \downarrow\right\rangle,
\end{aligned}
$$

where $\hat{U}_{\mathrm{RSB}}$ is the resonant red-sideband time-evolution operator that maps phonon-field information on two internal states of the ion, $\hat{R}(\pi / 2, \phi)$ is a spin rotation operator describing the $\pi / 2$ carrier pulse, with phase $\phi$ (taken here to be equal to zero), and $\left|\psi_{\downarrow}\right\rangle=\hat{R}^{\dagger}(\pi / 2,0)|\downarrow\rangle$. Similarly, $\left|\psi_{\uparrow}\right\rangle=$ $\hat{R}^{\dagger}(\pi / 2,0)|\uparrow\rangle$.

Equation (A1) implies that $P_{\downarrow}$ is the expectation value of $\left|\psi_{\downarrow}\right\rangle\left\langle\psi_{\downarrow}\right|$, with

$$
\left|\psi_{\downarrow}\right\rangle=\hat{R}^{\dagger}(\pi / 2,0)|\downarrow\rangle=(|\downarrow\rangle+|\uparrow\rangle) / \sqrt{2},
$$

in the state $|\Phi\rangle=\hat{U}_{\text {RSB }}|G \alpha, \downarrow\rangle$; similarly for $P_{\uparrow}$. Therefore, the contrast can be written as the expectation value of

$$
C=\langle\Phi|\hat{\Sigma}| \Phi\rangle, \quad \hat{\Sigma}=\left|\psi_{\downarrow}\right\rangle\left\langle\psi_{\downarrow}|-| \psi_{\uparrow}\right\rangle\left\langle\psi_{\uparrow}\right| .
$$

The fluctuations can be calculated from

$$
\left\langle\Phi\left|\hat{\Sigma}^{2}\right| \Phi\right\rangle-(\langle\Phi|\hat{\Sigma}| \Phi\rangle)^{2}=1-C^{2},
$$

and therefore the signal-to-noise ratio becomes

$$
\mathrm{SNR}=\frac{C \sqrt{\mathcal{N}}}{\sqrt{1-C^{2}}},
$$

where we have added the factor $\sqrt{\mathcal{N}}$ for the number of measurements. 
[1] H. A. Haus and J. A. Mullen, Quantum noise in linear amplifiers, Phys. Rev. 128, 2407 (1962).

[2] C. M. Caves, Quantum limits on noise in linear amplifiers, Phys. Rev. D 26, 1817 (1982).

[3] W. Wootters and W. Zurek, A single quantum cannot be cloned, Nature (London) 299, 802 (1982).

[4] D. Dieks, Communication by EPR devices, Phys. Lett. A 92, 271 (1982).

[5] V. Bužek and M. Hillery, Quantum copying: Beyond the nocloning theorem, Phys. Rev. A 54, 1844 (1996).

[6] T. C. Ralph and A. P. Lund, Nondeterministic noiseless linear amplification of quantum systems, in Ninth International Conference on Quantum Communication, Measurement and Computing (QCMC), edited by A. Lvovsky, AIP Conf. Proc. NO. 1110 (AIP, New York, 2009), p. 155.

[7] G. Y. Xiang et al., Noiseless linear amplification and distillation of entanglement, Nat. Photon. 4, 316 (2010).

[8] H. A. Haus, Electromagnetic Noise in Quantum Optical Measurements (Springer, New York, 2000).

[9] R. Kakarla, J. Schröder, and P. A. Andrekson, One photon-perbit receiver using near-noiseless phase-sensitive amplification, Light: Sci. Applic. 9, 153 (2020).

[10] S. C. Burd et al., Quantum amplification of mechanical oscillator motion, Science 364, 1163 (2019).

[11] D. Leibfried et al., Toward Heisenberg-limited spectroscopy with multiparticle entangled states, Science 304, 1476 (2004).

[12] M. Penasa, S. Gerlich, T. Rybarczyk, V. Metillon, M. Brune, J.M. Raimond, S. Haroche, L. Davidovich, and I. Dotsenko, Measurement of a microwave field amplitude beyond the standard quantum limit, Phys. Rev. A 94, 022313 (2016).

[13] R. Abbott et al., Observation of gravitational waves from two neutron star-black hole coalescences, ApJL 915, L5 (2021).

[14] V. Ménoret et al., Gravity measurements below $10^{-9} \mathrm{~g}$ with a transportable absolute quantum gravimeter, Sci. Rep. 8, 12300 (2018).

[15] D. Kramer, DARPA looks beyond GPS for positioning, navigating, and timing, Phys. Today 67(10), 23 (2014); D. Feng, Review of quantum navigation, IOP Conf. Ser.: Earth Environ. Sci. 237, 032027 (2019).

[16] Boulder Atomic Clock Optical Network (BACON) Collaboration, Frequency ratio measurements at 18-digit accuracy using an optical clock network, Nature (London) 591, 564 (2021).

[17] K. A. Gilmore et al., Quantum-enhanced sensing of displacements and electric fields with two-dimensional trapped-ion crystals, Science 373, 673 (2021).

[18] C. W. Helstrom, Quantum Detection and Estimation Theory (Academic Press, New York, 1976), Chap. VIII.4.

[19] A. S. Holevo, Probabilistic and Statistical Aspects of Quantum Theory (North-Holland, Amsterdam, 1982).

[20] S. L. Braunstein and C. M. Caves, Statistical Distance and the Geometry of Quantum States, Phys. Rev. Lett. 72, 3439 (1994).

[21] B. M. Escher, R. L. de Matos Filho, and L. Davidovich, General framework for estimating the ultimate precision limit in noisy quantum-enhanced metrology, Nat. Phys. 7, 406 (2011).

[22] B. M. Escher, L. Davidovich, N. Zagury, and R. L. de Matos Filho, Quantum Metrological Limits via a Variational Approach, Phys. Rev. Lett. 109, 190404 (2012).
[23] M. M. Taddei, B. M. Escher, L. Davidovich, and R. L. de Matos Filho, Quantum Speed Limit for Physical Processes, Phys. Rev. Lett. 110, 050402 (2013).

[24] C. L. Latune, B. M. Escher, R. L. de Matos Filho, and L. Davidovich, Quantum limit for the measurement of a classical force coupled to a noisy quantum-mechanical oscillator, Phys. Rev. A 88, 042112 (2013).

[25] J. Wang, L. Davidovich, and G. S. Agarwal, Quantum sensing of open systems: Estimation of damping constants and temperature, Phys. Rev. Res. 2, 033389 (2020).

[26] M. Kacprowicz, R. Demkowicz-Dobrzański, W. Wasilewski, K. Banaszek, and I. A. Walmsley, Experimental quantumenhanced estimation of a lossy phase shift, Nat. Photon. 4, 357 (2010).

[27] R. A. Fisher, On the dominance ratio, Proc. R. Soc. Edinburgh 42, 321 (1922).

[28] H. Cramér, Mathematical Methods of Statistics (Princeton University Press, Princeton, NJ, 1946).

[29] C. R. Rao, Information and the accuracy attainable in the estimation of statistical parameters, Bulletin Calcutta Math. Soc. 37, 81 (1945).

[30] K. C. MacCormick et al., Quantum-enhanced sensing of a single-ion mechanical oscillator, Nature (London) 572, 86 (2019).

[31] B. Yurke, S. L. McCall, and J. R. Klauder, SU(2) and SU(1, 1) interferometers, Phys. Rev. A 33, 4033 (1986).

[32] W. N. Plick, J. P. Dowling, and G. S. Agarwal, Coherent-lightboosted, sub-shot noise, quantum interferometry, New J. Phys. 12, 083014 (2010).

[33] J. Jing, C. Liu, Z. Zhou, Z. Y. Ou, and W. Zhang, Realization of a nonlinear interferometer with parametric amplifiers, Appl. Phys. Lett. 99, 011110 (2011).

[34] F Hudelist, J. Kong, C. Liu, J. Jing, Z. Y. Ou, and W. Zhang, Quantum metrology with parametric amplifier-based photon correlation interferometers, Nat. Commun. 5, 3049 (2014).

[35] B. E. Anderson, P. Gupta, B. L. Schmittberger, T. Horrom, C. Hermann-Avigliano, K. M. Jones, and P. D. Lett, Phase sensing beyond the standard quantum limit with a variation on the $\mathrm{SU}(1$, 1) interferometer, Optica 4, 752 (2017).

[36] D. Leibfried et al., Creation of a six-atom "Schrödinger cat" state, Nature (London) 438, 639 (2005).

[37] P. Groszkowski, H.-K. Lau, C. Leroux, L. C. G. Govia, and A. A. Clerk, Heisenberg-Limited Spin Squeezing via Bosonic Parametric Driving, Phys. Rev. Lett. 125, 203601 (2020).

[38] L. Pezze, A. Smerzi, M. K. Oberthaler, R. Schmied, and P. Treutlein, Quantum metrology with nonclassical states of atomic ensembles, Rev. Mod. Phys. 90, 035005 (2018).

[39] G. S. Agarwal, Quantum Optics (Cambridge University Press, Cambridge, 2013), Sec. 5.22.

[40] M. Kitagawa and M. Ueda, Squeezed spin states, Phys. Rev. A 47, 5138 (1993).

[41] G. S. Agarwal, R. R. Puri, and R. P. Singh, Atomic Schrödinger cat states, Phys. Rev. A 56, 2249 (1997).

[42] C. Song et al., Observation of multi-component atomic Schrödinger cat states of up to 20 qubits, Science 365, 574 (2019). 